\title{
Microexplosions polysterene microparticles on substrate covered by aluminum
}

\author{
V.S. Vasilev ${ }^{1,2}$, R.V. Skidanov ${ }^{1,2}$ \\ ${ }^{1}$ Samara National Research University, 34 Moskovskoe Shosse, 443086, Samara, Russia \\ ${ }^{2}$ Image Processing Systems Institute - Branch of the Federal Scientific Research Centre "Crystallography and Photonics" of Russian Academy of Sciences, \\ 151 Molodogvardeyskaya st., 443001, Samara, Russia
}

\begin{abstract}
Annotation
Experimental results of microexplosion polysterene microparticles diameter equals 5 micron located on substrate covered by aluminum layer thickness in 100 nanometers were showed. Produced a comparison results of experiment on quartz substrate and on aluminum substrate. As a source of radiation was chosen a laser with wavelength equals 355 nanometers.
\end{abstract}

Keywords: microexplosions; ultraviolet beam, polysterene microbits; velocity of microparticles; quartz substrate; aluminum substrate

\section{Introduction}

Currently, all manipulation works are now heading for reducing the size of the moving objects. There are plenty of microparticles manipulation methods using optical traps of different types. However, it is desirable to have a method to move a relatively large micro-objects. Especially such objects could be found in biological research (sporules, microslides of tissue, large cells). And it is necessary to prevent an effect even of a minimum quantity of light radiation on the biological micro-object. It is possible to carry out mechanical micro-manipulation by means of mechanical micro tweezers. This method is invasive for microparticles manipulating.

Typical sizes of the roaming micro-objects using common optical trap are from fractions of a micrometer to about ten micrometers. It is necessary to significantly increase the power of the light beam with the increase of the micro-objects size. Because anyway some fraction of the light beam energy is absorbed by the object, there is a certain limit for the microparticles size which can be moved by the forces of optical trapping. The precise value of this size depends on many parameters: the micro-objects absorption coefficient, fluid properties, micro-object surface shape, etc. A rough estimate of this size for transparent spherical micro-objects gives a value of about $30 \mu \mathrm{m}$. It should be noted that the micro-object with a size close to the limit is experienced strong thermal impact. Things get worse with the movement of opaque micro-objects in the light traps. The limiting size is reduced by one and a half to two times. At the same time, the micro-objects with sizes up to 100 microns are still quite small for a mechanical movement. There is another complicate combined method of micro-objects grasping with the usage of light and ultrasound [5]. But here is another [6] described simpler method of moving micro-objects by means of the microexplosions of polystyrene microparticles in a beam of an ultraviolet laser with a wavelength of $355 \mathrm{~nm}$.

In this paper we will consider a method of moving micro-objects with explosion of polystyrene microparticles on a substrate coated with a layer of aluminium. As compared with microexplosions on a quartz substrate, this method significantly reduces expended energy of microexplosions due to the interference of the beam, which is incident on the substrate and a beam reflected from the surface of the substrate.Object of study in this research is calculation average velocity polysterene microparticles with diameter equals 5 micron after microexplosion occurring under the pressure of laser emission with wavelength 355 nanometers.

The subject of the study is the behavior of polystyrene micro-particles in the explosion.

The aim of this work is the experimental test of interference effect between incident and reflected beams in the explosion on a substrate covered with aluminum. Another aim of this work is the calculation of scattering speed of microparticles after the explosion and finding the parameters for explosion occurrence.

In accordance with the intended aims following tasks were summarized:

- in situ observation of polystyrene microparticles microexplosion on a substrate covered with aluminum under the action of ultraviolet laser with a wavelength of $355 \mathrm{~nm}$;

- the calculation of the average dispersion velocity of polystyrene microparticles after the explosion;

- finding the system parameters for the observed microexplosions of polystyrene microspheres. Scientific and practical novelty and significance of the results:

- full-scale experiment with the microexplosion of polystyrene microparticles on a substrate covered with aluminum under the action of an ultraviolet laser with a wavelength of $355 \mathrm{~nm}$ was successfully completed.

- the average expansion rate of polystyrene microparticles after microexplosion was calculated.

- the system parameters for the observed polystyrene microspheres microexplosions were found.

\section{Theoretical description observed effect}

In this experiment, there is an effect of interference of incident and reflected waves. As a result of usage a single source of light radiation the incident and reflected waves are coherent. Thus, the total intensity of incident and reflected waves [7] can be represented as follows: 
where $I_{1}, I_{2}$ - the intensity of the incident and reflected beams, respectively, $\delta$ - the phase difference between these beams. Considering that quartz glass has a transmittance of $99 \%$ and aluminum reflects about $93-94 \%$, it is possible to write the following:

$I_{1}>I_{2}$

Then, inserting into the formula above, we get that $\mathrm{I}_{\max }=4 \mathrm{I}_{1}$ and $\mathrm{I}_{\min }=0$. This formula will be tested experimentally - if the microexplosions will occur on the aluminum substrate at the power level at which microexplosions of polystyrene microparticles on a quartz substrate were not observed, so it is possible to draw a conclusion about the strengthening of the two beams through their interference.

\section{Experiment}

Consider the installation, which was used in the observation process of the full-scale experiment with microexplosion of polystyrene microparticles on a substrate coated with aluminium in thickness of $100 \mathrm{~nm}$. The application of the aluminium substrate was made with the use of plant «Carolina D 12 A» designed for magnetron sputtering on ceramic, silicon and other substrates with sizes up to $100 \mathrm{~mm}$. The following keys were added to the optical diagram in figure 1: 1 - continuous UV laser DTL - 375 with wavelength $355 \mathrm{~nm}$ and maximal average power equals to $40 \mathrm{mV} \mathrm{[4];} \mathrm{2,3} \mathrm{-} \mathrm{rotary} \mathrm{mirrors;} 4$ - semitransparent cubic; 5 - focused microobjective $(20 \mathrm{x}) ; 6$ - substrate covered by aluminum with polysterene microparticles; 7 - CCD - camera FastVideo 500 E with resolution 640x480.

Now let's move to the description of the experiment. The light by means of rotary mirrors and microscope objective is focused into the required area of the substrate with microparticles. In view of the high reflection coefficient of aluminium (about 93-94\%) the process of microexplosions on the aluminium substrate needed to be monitored in the reflected light. For separation of the incident and the reflected beams it is used a cube with translucent mirrors. Reflected light falls on the camera and the resulting image is processed on the computer.

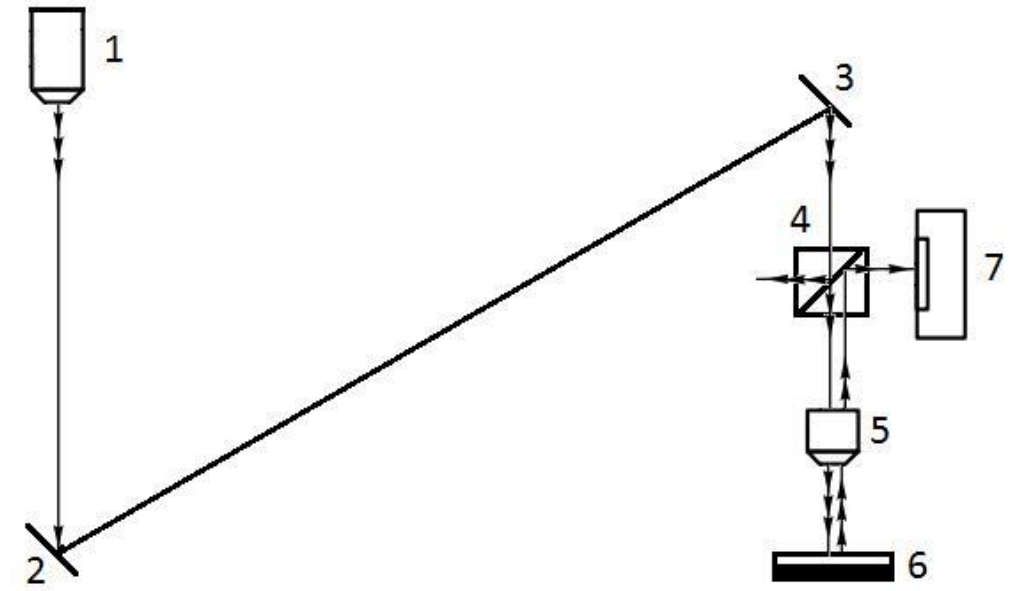

Fig.1. Optical setup for microexplosions polysterene microparticles on substrate covered by aluminum.

Let's turn to the experimental results. The original experiment took place at the maximum value of laser radiation power obtained with the pulse frequency of $3000 \mathrm{~Hz}$. But with this value there was a destruction of the substrate surface covered with aluminum. Consequently, the beam power was reduced to a value of $6.17 \mathrm{~kW}$, which stops the observed damage to the surface of the substrate. Thus, it minimized the influence of melting and destruction of the substrate surface on the movement of microparticles of polystyrene.

An experiment was carried out with deposition of polystyrene microparticles floating in the water on the substrate covered with aluminum. As the result, it was obtained a footage of polystyrene microparticles movement after the explosion, which is achieved by superposition of the incident and reflected beams of laser radiation. Now we will explain how we made these conclusions.

Firstly, in order to eliminate the influence of melting and destruction of the substrate surface covered with aluminium on the movement of polystyrene particles, the particles were inputted to the surface in the water. Thus, the water absorbs the portion of energy that came from breaking the surface of the substrate.

Given that the light almost does not pass through the surface covered with aluminum, then all the power goes into heating the surface (this effect was eliminated due to the choice of required capacity and the introduction of particles in solution with water), and the remaining part is reflected (about 93\%). We find that in the explosion of polystyrene microparticles affects only the energy of the incident beam and the energy of the reflected beam.

In order to see whether the incident and reflected beams interact with each other or the main contribution is made only by the incident beam, another experiment was carried out which eliminates these issues. The substrate covered with aluminium was replaced by a quartz substrate with the transmittance of about $99 \%$. Thus, if the former power of the laser radiation the microexplosion of polystyrene microparticles will not occur it turns out that this reflected beam interacts with the incident beam in a certain way. 
After conducting full-scale experiments on a substrate covered with aluminum, there was an explosion of polystyrene microparticles, in which a displacement of nearby microparticles of polystyrene occurred. In case of changing of substrate coated with aluminium on a quartz substrate and leaving unchanged all the parameters of the scheme the explosion of polystyrene particles was not observed. As the result, we can conclude that explosion of polystyrene microparticles occurs on a substrate covered with aluminum due to the interaction of the incident and reflected beams.

It was also calculated the average rate of expansion of the polystyrene particles located on a substrate coated with aluminium after the explosion of a nearby polystyrene particles. This value is $0.77 \mathrm{~mm} / \mathrm{s}$.

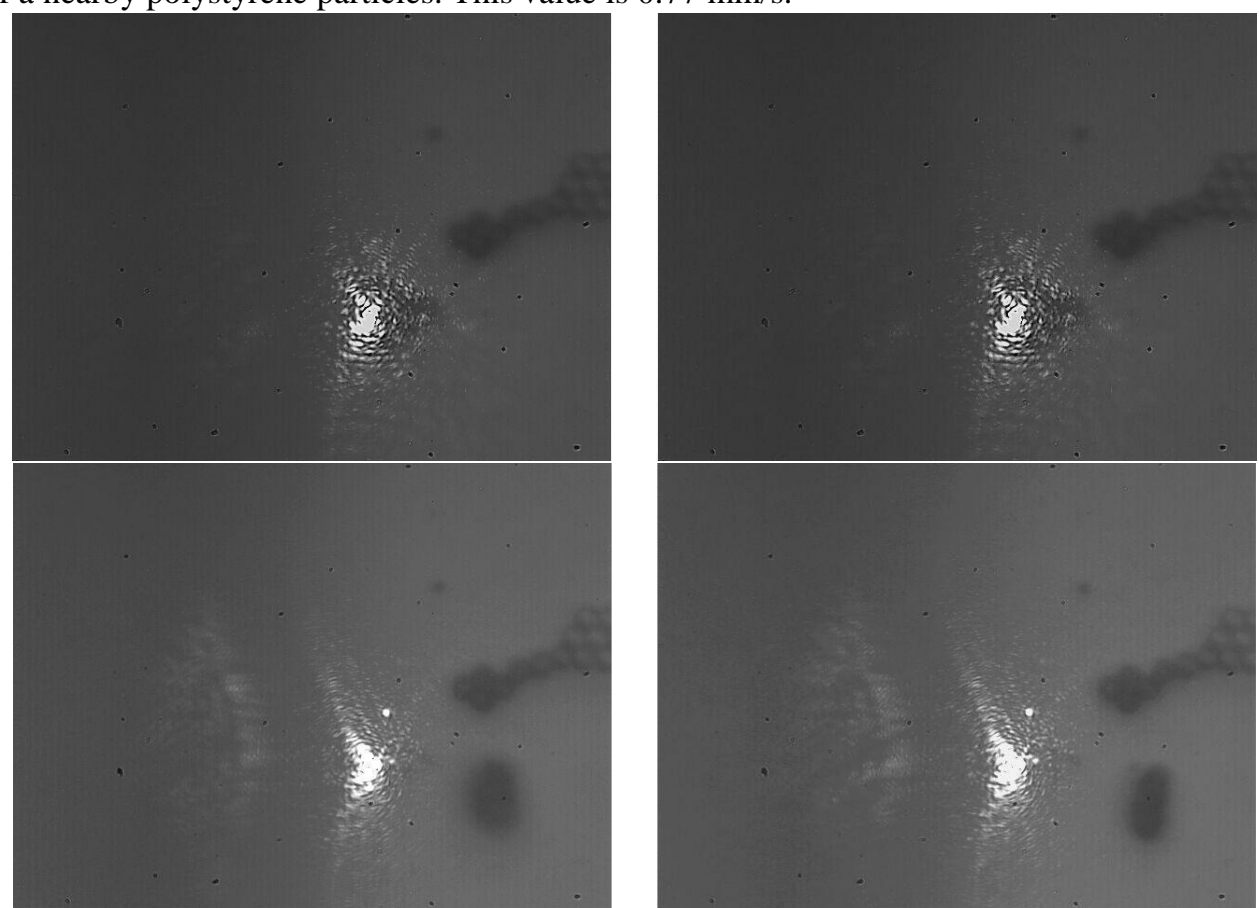

Fig.2. Results of the experiment on explosions polysterene microparticles using substrate covered by aluminum. Time interval between frames is equal to 10 $\mathrm{ms}$.
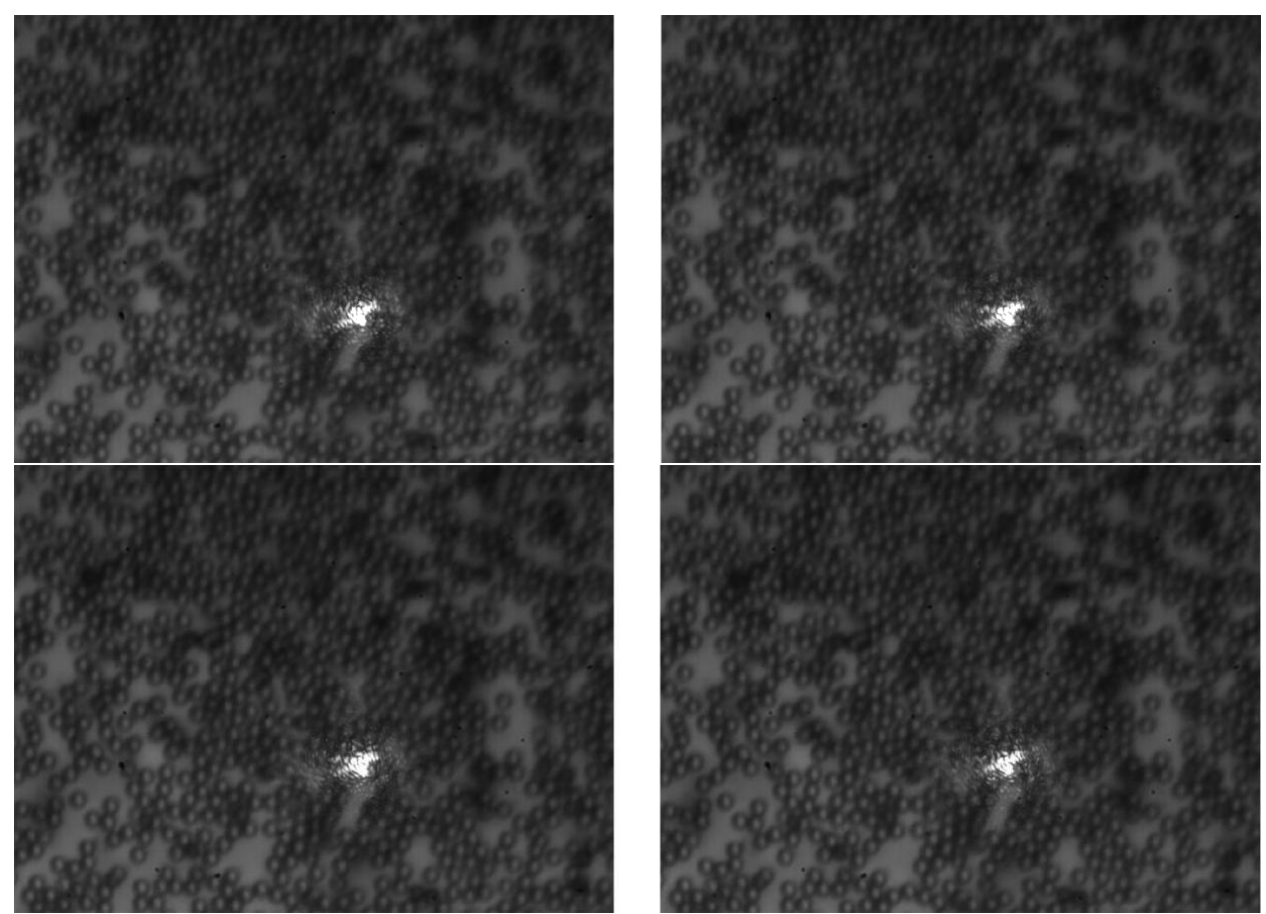

Fig.3. Results of the experiment on explosions polysterene microparticles using quartz substrate. Time interval between frames is equal to $10 \mathrm{~ms}$.

\section{Conclusion}

In the process of performing this work the following results were obtained:

1) An experiment was conducted, in which we discovered the existence of the interference between incident and reflected waves using aluminum substrate.

2) The expansion velocity of the particles using a 20x focusing microobjective was experimentally calculated. The speed value is $0.77 \mathrm{~mm} / \mathrm{s}$;

3) The parameters of explosion occurrence were found. The parameter is the average radiation power. The explosion occurs when values are higher than $6.17 \mathrm{~kW}$. 


\section{Acknowledgements}

The work was partially funded by the RF Ministry of Science and Education as part of state-assigned task No. 3.3025.2017/PCh and by the Russian Foundation for Basic Research (RFBR) (grants Nos. 16-29-11744 ofi_m and 16-29-09528 ofi_m).

\section{References}

[1] Zemranek P, Jonras A, Srramek L, Liska M. Optical trapping of nanoparticles and microparticles by a Gaussian standing wave. Optics Letters 1999; 24(21): $1448-1450$.

[2] De AK, Roy D, Dutta A, Goswami D. Stable optical trapping of latex nanoparticles with ultrashort pulsed illumination. Applied Optics 2009; 48(31): 3337.

[3] Bosanac L, Aabo T, Bendix PM, Oddershede LB. Efficient Optical Trapping and Visualization of Silver Nanoparticles. Nano Letters 2008; 8(5): 14861491.

[4] Khonina SN, Kotlyar VV, Skidanov RV, Soifer VA. Diffraction optical elements for oprical manipulation of microparticles. Official materials scientific and practical conference "Holography in Russia and abroad. Science and practice" 2004; 2(4): 62.

[5] Thalhammer G, Steiger R, Meinschad M, Hill M, Bernet S, Ritsch-Marte M. Combined acoustic and optical trapping. Biomedical Optics Express 2011; 2(10): 2859-2870.

[6] Skidanov RV, Morozov AA, Porfirev AP. Composite light beam and microexplosion for optical micromanipulation. Computers Optics 2010;34(3): 371375.

[7] Sivuhin DV. Course of general physics V. 4. Optics. M.: Science, 1980.

[8] Bardin AN. Assembly and alignment optical devices. M.: Higher school, 1968. 